\title{
Escurrimiento en pavimentos de bloques de suelo-cemento: un abordaje experimental
}

\section{Runoff on Pavements of Soil-Cement Blocks - an Experimental Boarding}

\author{
Zegarra-Tarqui Jorge Luis \\ Escuela de Ingeniería \\ Universidad Federal de Minas Gerais, Brasil \\ Correo: jlztarqui@yahoo.com.br \\ Santos-de Brito Jeferson \\ Produman Engenharia, Brasil \\ Correo:jeferson.brito.eng@gmail.com
}

\author{
De Fátima-Carvalho Miriam \\ Escuela de Ingeniería \\ Universidad Católica de Salvador, Brasil \\ Correo:miriam@ucsal.br
}

Información del artículo: recibido: abril de 2013, aceptado: febrero de 2014

\begin{abstract}
Resumen
El artículo evalúa la reducción del escurrimiento superficial en pavimentos construidos con bloques rectangulares de suelo-cemento. Los ensayos fueron realizados en un pavimento piloto con bloques de suelo-cemento, asentados en una caja metálica de $50 \mathrm{~cm} \times 50 \mathrm{~cm}$ (área $=2500 \mathrm{~cm}^{2}$ ), con pendientes de 1,3 y $5 \%$. Se utilizaron intensidades medias de $76.9 \mathrm{~mm} / \mathrm{h}$ y $117.7 \mathrm{~mm} / \mathrm{h}$, valores próximos a las intensidades calculadas por la curva intensidad-duración-frecuencia (i-d-f) de la ciudad del Salvador, Brasil, para tiempo de retorno de 2 y 5 años, respectivamente. El coeficiente de escurrimiento medio fue $C=0.61$, este valor es cercano al coeficiente de pavimento con bloques rectangulares $(C=0.6)$ y es inferior al valor del coeficiente de pavimento de bloque de concreto $(C=0.78)$. Por otro lado, si consideramos que las áreas mayores que $2500 \mathrm{~cm}^{2}$ son compuestas por el acoplamiento de unidades de área de $50 \times 50$ centímetros, entonces, las pérdidas por salpicamiento forman parte del escurrimiento superficial, obteniendo el coeficiente $\mathrm{C}_{\text {superficialtsalpicamiento }}$ el cual presentó valores en la faja de 0.74 a 0.89 ; estos valores se encuentran cerca del coeficiente de pavimento de bloque de cemento $(C=0.78)$ y son inferiores al de pavimento de concreto $(C=0.95)$, pero considerando factores como tiempo de desplazamiento del escurrimiento sobre la superficie, depresiones en la superficie, evaporación y rugosidad del pavimento, entre otros, este valor debe disminuir. Entonces, el pavimento de bloques de suelo-cemento puede considerarse dentro de la categoría de semipermeable para las dimensiones de área utilizadas.
\end{abstract}

\section{Descriptores:}

- hidrología urbana

- coeficiente de escurrimiento superficial

- pavimento permeable

- bloque suelo-cemento 


\begin{abstract}
The article evaluates the reduction of runoff in pavements constructed with rectangular blocks of soil-cement. The tests were conducted in a pilot pavement built with soil-cement blocks, seated in a metal box of $50 \mathrm{~cm} \times 50 \mathrm{~cm}$ (area $=2500 \mathrm{~cm} 2)$, with declivities of 1\%,3\% and 5\%. Mean intensities of $76.9 \mathrm{~mm} / \mathrm{I} 117.7 \mathrm{~mm} / \mathrm{h}$ were used, values close to the intensities calculated by intensity-frequency-duration (i-f-d) equation of the city of Salvador, Brazil, for return periods of 2 and 5 years, respectively. The medium runoff coefficient was $C=0.61$, this value is close to the coefficient of pavement with rectangular blocks $(C=0.6)$ and it has a lower value than the coefficient for concrete block pavement $(C=0.78)$. On the other hand, considering that areas with more than $2500 \mathrm{~cm}^{2}$ are constituted by coupling of area units of $50 \mathrm{~cm} \times 50 \mathrm{~cm}$, the splash losses are part of the runoff, obtaining the coefficient $C_{\text {superficial + splashing' }}$ which showed values in the 0.74 to 0.89 range, these values were found close to the coefficient of concrete block pavement $(C=0.78)$ and below the concrete pavement $(C=0.95)$, respectively, but factors such as displacement time of runoff on surface, depressions on the surface, roughness of pavement, evaporation and others, should reduce this value. Then, the pavement of soil-cement blocks can be considered in the category of semi-permeable for the area size used.
\end{abstract}

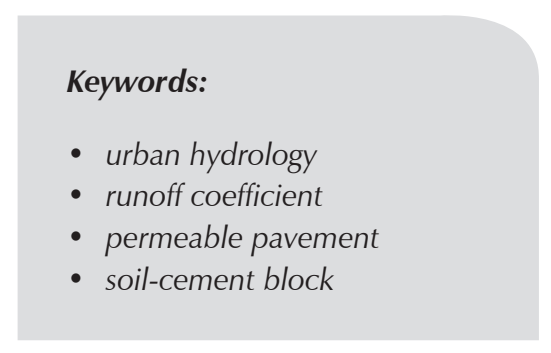

\section{Introducción}

La ocupación del suelo en el medio urbano se caracteriza por el aumento del área impermeable ocasionando, principalmente, el aumento del escurrimiento superficial (crecidas), la degradación de la calidad de las aguas urbanas y la disminución de la recarga de las aguas subterráneas. De forma general, en las ciudades brasileiras la cuestión relativa al drenaje urbano ha sido tratada, de forma cronológica, desde tres puntos de vista. Según Silveira (1998), inicialmente prevaleció el concepto higienista, fundamentado en la salud pública de las áreas urbanas, preconizado por el drenaje rápido de las aguas pluviales mediante canalizaciones. Surgen las primeras ecuaciones de la cuantificación de la precipitación y del escurrimiento superficial para el dimensionamiento hidráulico de las obras de drenaje. En seguida, vino el enfoque de racionalización y normalización de los cálculos hidrológicos. Permanece el concepto de drenaje rápido, pero se establece un mejor cálculo hidrológico para dimensionamiento de las obras hidráulicas, surgiendo el método racional y de la normalización de los cálculos. La tercera y más actual, es un enfoque científico y ambiental del ciclo hidrológico urbano que estableció la hidrología urbana de hoy, las acciones (estructurales y no estructurales) se vuelcan para mantenerlas condiciones deescurrimientodepre-urbanización actuando al nivel local, en la fuente de generación del mismo. Una propuesta es la utilización de dispositivos de regulación del drenaje, en ese contexto, los pavimen- tos permeables son dispositivos que reducen el pico del escurrimiento superficial, aumentan la infiltración y la evaporación (Andersen et al., 1999). James y Von (2003) y Stormwater (2011) mencionan que los pavimentos permeables reducen la carga de sedimentos y remueven materiales pesados y nutrientes del escurrimiento (superficial y percolado). La reducción de la eficiencia del dispositivo está relacionada con la intensidad del tránsito sometido y la periodicidad del mantenimiento (limpieza o sustitución del material agregado) a fin de evitar entupimiento por material fino y reducir la capacidad de infiltración y remoción.

Según Hunt y Collins (2008) los pavimentos permeables están compuestos por una capa superficial (con el tipo de pavimento permeable empleado), una capa de arena para asentamiento y dos camadas (base y sub-base) compuestas por agregados.

Existen cinco tipos de pavimentos: de asfalto poroso, de concreto poroso, de bloques huecos de concreto rellenados de material granular (arena) o vegetación rastrera (grama), de bloques de concreto o piedra colocados en padrón intertrabado y de grilla con estructura de plástico. Según Marchioni y Silva (2010), los pavimentos intertrabados de bloques se asientan sobre un conjunto de capas denominadas de capa de asentamiento, base, sub-base y sub-lecho y pueden utilizarse tubos de drenaje cuando el suelo local tenga baja permeabilidad (figuras 1 y 1a). La función de estas capas es facilitar la infiltración en el suelo (sublecho) y funcionar como reservorios de detención del agua. 


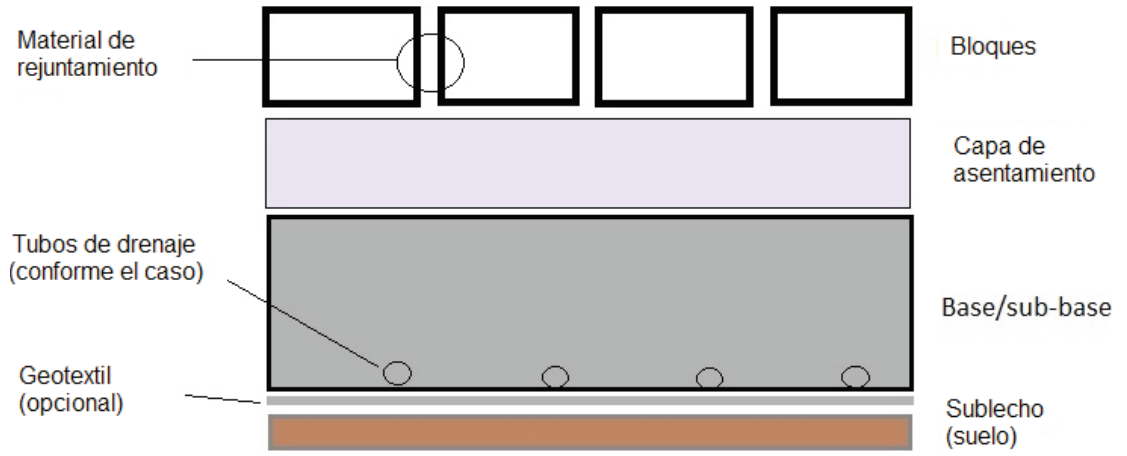

Figura 1. Perfil padrón de un pavimento de bloques intertrabados
Araujo et al. (2000) consideran como pavimentos semipermeables los bloques de concreto o piedra con juntas de arena, los otros tipos de pavimento mencionados se consideran permeables. Para la subdivisión se toma como criterio el área disponible para infiltración, que en el caso de los bloques está constituida por el área de las juntas, que representan entre 5 y $10 \%$ del área de la parcela del pavimento, conforme el tipo de bloque, ya para los otros tipos de pavimentos (bloques con huecos y grillas de plástico) el área disponible para infiltración es superior. Los tipos de bloques más comunes son bloques de granito, bloques de concreto industrializado y bloques de suelo-cemento, en la figura 2 se muestran ejemplos de pavimentos. La investigación se concentra en el estudio de bloques rectangulares de suelo-cemento.

Para evaluar la eficiencia del pavimento (capacidad de reducir el caudal pico y favorecer la infiltración) se emplea el coeficiente de escurrimiento (C), definido como la razón entre los volúmenes escurrido y precipitado. En régimen permanente, el coeficiente varía en la faja de 0 a 1 en condiciones naturales, por ejemplo en la vegetación nativa, el coeficiente tendría un valor 0.35 , entonces se busca el uso de pavimentos y otro tipo de dispositivos que reduzcan el volumen escurrido, con la finalidad de aproximar el valor real del coeficiente de escurrimiento para el valor de coeficiente de pre-urbanización. En el caso específico del pavimento semipermeable el coeficiente de escurrimiento depende del tipo de material del bloque, el espacio entre las juntas, la rugosidad del bloque, la irregularidad de la superficie (depresiones), la pendiente, el tamaño del área pavimentada, los factores de forma del área (alargado, redondeado, rectangular, etcétera), el material utilizado en el asentamiento de los bloques y del tamaño de las capas empleadas en el asentamiento.

En la tabla 1 se presentan datos experimentales, así como el coeficiente de escurrimiento para algunos tipos de pavimentos evaluados para una parcela de $1 \mathrm{~m}^{2} \mathrm{de}$ área (Araújo et al., 2000) y pendientes menores que $5 \%$.

En la tabla, el coeficiente de escurrimiento para pavimento de paralelepípedo es inferior al de los bloques de concreto, los autores atribuyen esta diferencia a los siguientes factores: el área de la junta (fracción de área del pavimento reservado para la holgura -juntaentre los bloques), ya que un área mayor favorece la

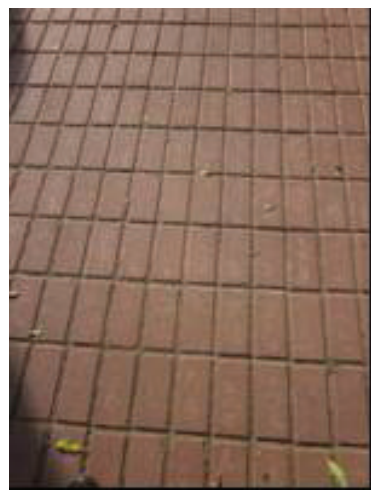

a)

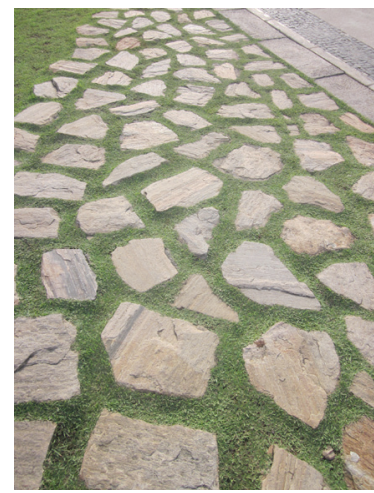

b)

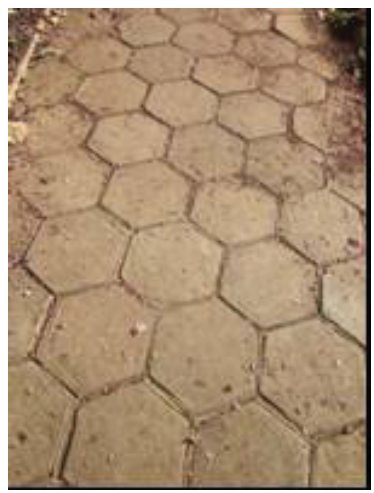

c)

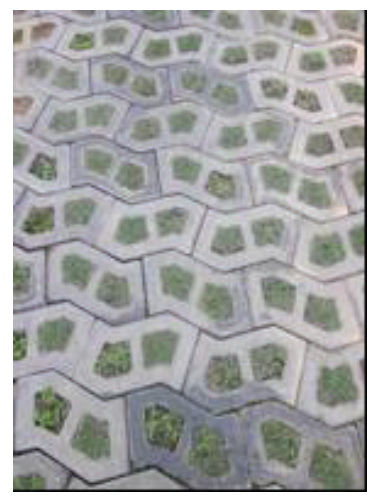

d)

Figura 1a. Pavimentos con: a) bloque rectangular de concreto, b) bloque irregular de piedra, c) bloque hexagonal de concreto y d) bloque de concreto con hueco 
Tabla 1. Coeficiente de escurrimiento para diferentes tipos de pavimento

\begin{tabular}{lccccc}
\hline \multicolumn{1}{c}{ Tipo de pavimento } & $\begin{array}{c}\text { Suelo } \\
\text { compactado }\end{array}$ & Concreto & $\begin{array}{c}\text { Bloque de concreto no } \\
\text { rectangular }\end{array}$ & $\begin{array}{c}\text { Bloque } \\
\text { rectangular de piedra }\end{array}$ & $\begin{array}{c}\text { Bloque } \\
\text { hueco }\end{array}$ \\
\hline Fecha & $03 / 06 / 98$ & $28 / 10 / 98$ & $29 / 07 / 98$ & $13 / 10 / 98$ & $27 / 01 / 99$ \\
Hora de inicio & $14: 06$ & $15: 15$ & $15: 20$ & $11: 20$ & $10: 08$ \\
Intensidad $(\mathrm{mm} / \mathrm{h})$ & 112 & 110 & 116 & 110 & 110 \\
Lluvia total $(\mathrm{mm})$ & 18.66 & 18.33 & 19.33 & 18.33 & 10.99 \\
Escurrimiento total $(\mathrm{mm})$ & 12.32 & 17.45 & 15.00 & 0.60 & 0.5 \\
Coeficiente de escurrimiento & 0.66 & 0.95 & 0.78 & 32.72 & 0.03 \\
Humedad inicial del suelo $\left(\mathrm{cm}^{3} / \mathrm{cm}^{3}\right)$ & 32.81 & 32.73 & 32.71 & 32.24 \\
\hline
\end{tabular}

Fuente: Araújo et al. (2000)

infiltración y reduce el coeficiente de escurrimiento; el asentamiento irregular de los paralelepípedos favorece la formación de depresiones en la superficie y, en consecuencia, se tiene un aumento en la capacidad de retención y las pérdidas por infiltración en las bordas que cercan el área de estudio; entonces al hacer el balance hídrico la parcela de infiltración tendría un valor levemente sobreestimado, pues las pérdidas sería atribuidas a la verdadera infiltración. Otro factor a considerar es la pérdida por salpicamiento en las laterales de la parcela experimental, cuya evaluación es difícil en investigaciones de campo y tiene un papel importante en la sobreestimación de la infiltración para parcelas experimentales pequeñas $\mathrm{y}$, por ende, en la subestimación del coeficiente de escurrimiento para el pavimento empleado.

Para este estudio se construyó una parcela experimental en laboratorio, que permitió describir y cuantificar los flujos por infiltración y escurrimiento superficial, así como evaluar las pérdidas por salpicamiento en una parcela experimental de pequeño porte y su influencia en la subestimación del coeficiente de escurrimiento. El uso de suelo-cemento como material para la confección de bloques de pavimento en áreas urbanas de baja renta es una propuesta interesante debido al bajo costo del material utilizado, la fabricación artesanal y la participación de mano de obra local.

\section{Procedimiento experimental}

Definición de la intensidad y pendiente

Para el cálculo de la intensidad de lluvia se utilizó la ecuación de la curva de intensidad-duración-frecuencia (i-d-f) de la ciudad de Salvador, Estado de Bahia, Brasil, propuesta por Matos (2006) válida para un tiempo de retorno de hasta 10 años, lo suficiente para su utilización en proyectos de micro drenaje (ecuación 1).

$$
i=\frac{157.861 * T^{0.2443}}{(t+22)^{0.94 * T^{-0.0355}}}
$$

donde

$i=$ intensidad de la lluvia de proyecto, en $\mathrm{mm} / \mathrm{h}$

$T=$ período de retorno en años: tiempo medio en el cual una precipitación dada puede ser igualada o superada por lo menos una vez

$t=$ duración de la lluvia de proyecto, en minutos.

A partir de la ecuación 1 se obtuvo una intensidad de lluvia calculada de $77.9 \mathrm{~mm} / \mathrm{h}$ cuando se empleó $t=10$ $\min$ y $T=2$ años y para situaciones más extremas se encontró intensidad de $107.8 \mathrm{~mm} / \mathrm{h}$ con $t=10 \mathrm{~min}$ y $T=$ 5 años.

Fueron adoptadas pendientes de 1, 3 y 5\%, similares a las utilizadas por Araújo et al. (2000), con la finalidad de establecer comparaciones del coeficiente $\mathrm{C}$ y evaluar la eficiencia del pavimento estudiado.

\section{Pavimento de suelo-cemento}

Se construyeron bloques de suelo-cemento con proporción de 1:20, de la siguiente forma: primero el suelo se tamizó después de secar al aire libre, enseguida se utilizó para mezclar el suelo con el cemento una mezcladora de tambor, después de homogeneizado el material pasó por un tamiz y se moldeó con ayuda de una prensa manual. Los bloques a los 28 días alcanzaron una resistencia media de $10 \mathrm{MPa}$ y una absorción media de $15 \%$ conforme lo presentado en el trabajo de Brandão (2010).

Experimentalmente, la inserción de todas las capas (figura 1) en la confección de la caja experimental representaría una profundidad mayor que la altura de los bloques, por consecuencia, el conjunto -caja experi- 
mental, bloques y capas- tendría un peso considerable haciendo el experimento inviable, entonces se definió solo considerar los bloques y la capa de asentamiento de arena, este conjunto fue denominado: conjunto bloque-arena.

Para la instalación del pavimento se confeccionó una caja de drenaje de fierro galvanizado, con pintura de protección, dimensionada de forma que soportara el peso de los bloques, de la capa de arena y del geotextil, en total $70 \mathrm{~kg}$. La caja metálica posee dimensiones de 50 $\times 50 \times 10 \mathrm{~cm}$ (ancho $\times$ largo $\times$ alto). Un tubo de drenaje en la base de la caja permitió medir el escurrimiento subsuperficial y una canaleta colectora en la parte superior permitió medir el escurrimiento superficial del pavimento. Ambos dispositivos se conectaron a mangueras y se direccionaron hacia frascos de medición de volumen.

Los bloques de suelo-cemento se asentaron sobre una capa de arena con $2 \mathrm{~cm}$ de espesor, (figura 2a), sobrepuesta a una manta geotextil para evitar la pérdida del material granular por el tubo de drenaje en la base. El trabamiento de los bloques fue del tipo trama (figura 2c), con juntas de $5 \mathrm{~mm}$ llenas de arena, entonces el área destinada a las juntas tenía $200 \mathrm{~cm}^{2}$ que representan aproximadamente $8 \%$ del área total de la caja de ensayo. La figura $2 \mathrm{~b}$ muestra una vista del pavimento de ensayo.

\section{Proceso de simulación experimental}

El simulador de lluvia de disco giratorio (modelo FEL 3, fabricado por la Armfield) usado en este estudio se compone de una cámara pulverizadora soportada por estructura metálica y accesorios que comprenden reservorio de agua, bomba de recalque y panel de control (Armfield, 1998). El equipo funciona mediante bombeo de agua del reservorio hasta la cámara pulverizadora, pasando por una válvula de gaveta, que permite ajustar la presión deseada, a través del conocimiento de la presión del agua que llega al bocal por medio del manómetro de Bourbon. Luego, abajo del bocal, el chorro de agua es interceptado por un disco giratorio, que tiene su velocidad de rotación ajustada y calibrada en el panel de control que logra $240 \mathrm{rev} / \mathrm{min}$ y su abertura varía de $5^{\circ}$ a $40^{\circ}$, de $5^{\circ}$ en $5^{\circ}$, permitiendo obtener variaciones en las intensidades de las precipitaciones inducidas. La figura $2 \mathrm{~b}$ ilustra el simulador de lluvia utilizado en la investigación.

Se calibró el simulador para las intensidades de precipitación deseadas, $77.9 \mathrm{~mm} / \mathrm{h}$ y $107.8 \mathrm{~mm} / \mathrm{h}$, ajustando la abertura del disco, la presión del agua, la velocidad de rotación del disco y la posición de la mesa metálica en el interior de la cámara pulverizadora. Usando la curva de calibración del simulador realizada por Cardoso (2007), las intensidades más próximas a las deseadas fueron $76.9 \mathrm{~mm} / \mathrm{h}$ con abertura de $5^{\circ}$ y presión 0.6 bar y $117.7 \mathrm{~mm} / \mathrm{h}$ con abertura de $10^{\circ}$ y presión 0.4 bar.

Las pruebas preliminares en el pavimento permitieron definir los tiempos del inicio del escurrimiento superficial y subsuperficial, de la estabilización de los escurrimientos y del intervalo de colecta.

A partir de la aplicación de la formulación para volumen de control en la ecuación básica de la conservación de la masa fue posible analizar el proceso dinámico (etapas) de los escurrimientos: superficial, salpicamiento, infiltración y subsuperficial. La denominación de

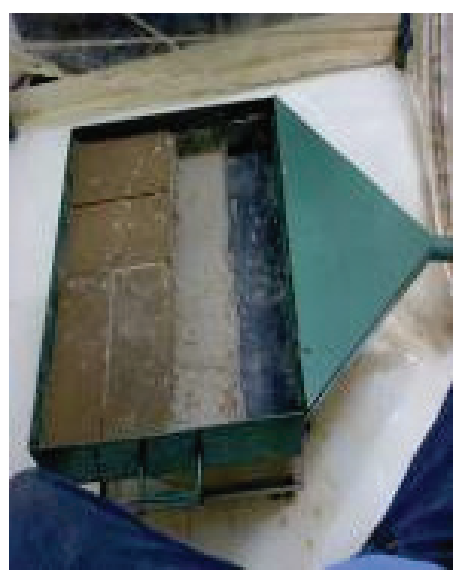

a)

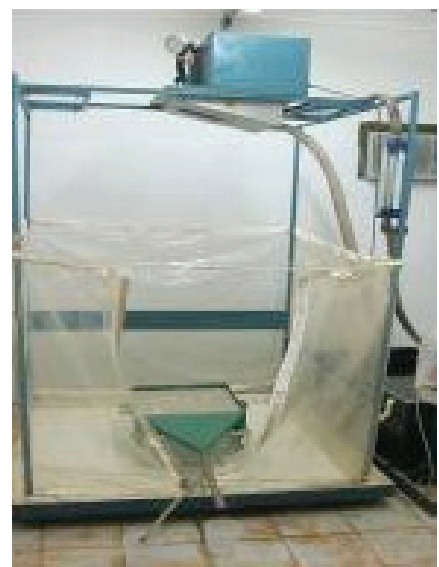

b)

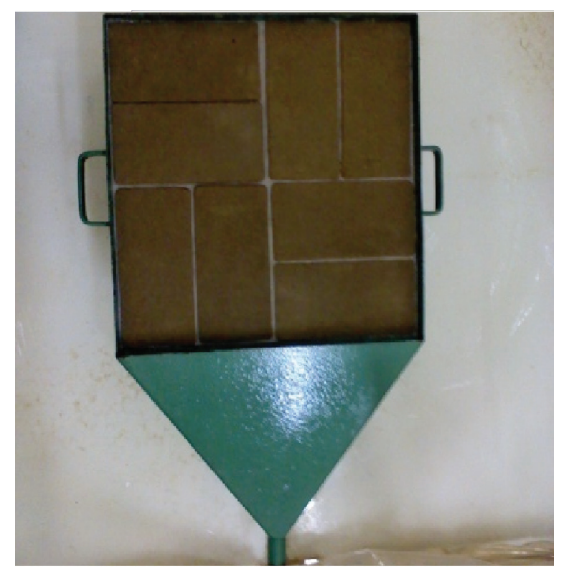

C)

Figura 2. Capas del pavimento y montaje del ensayo, a) montaje de los bloques con la capa de asentamiento de arena, b) vista frontal del simulador y la caja de ensayos, c) detalle del trabamiento de los bloques tipo trama en la caja de ensayos 


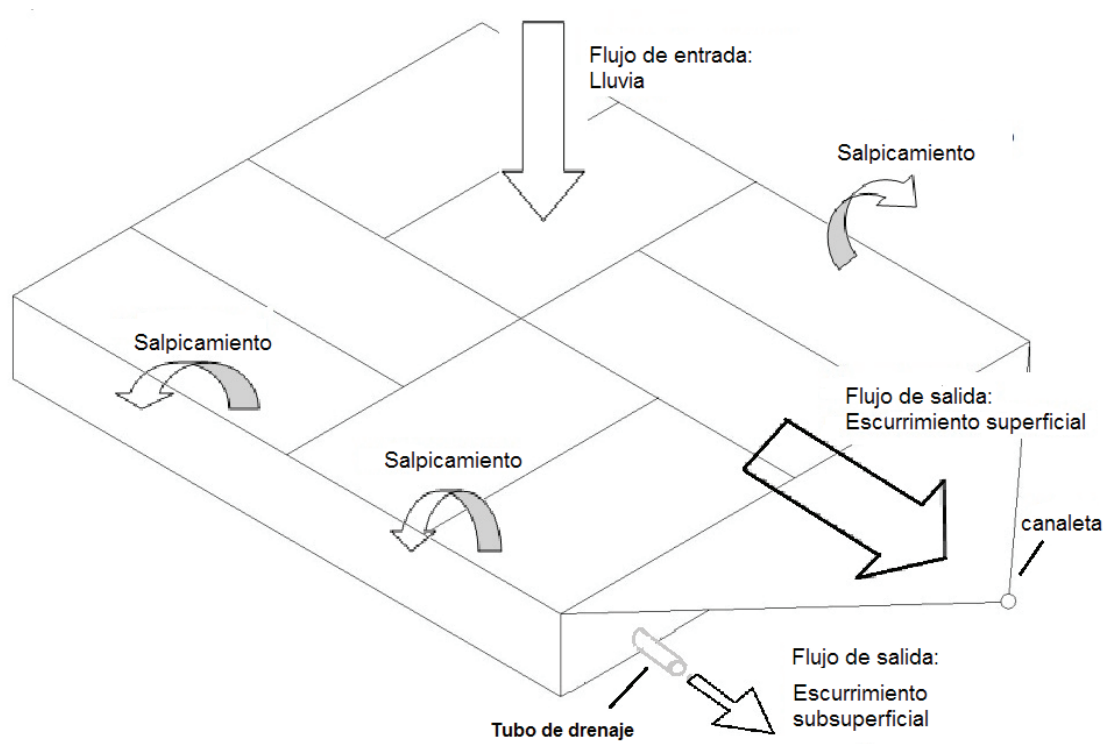

Figura 3. Esquema de pérdida del agua por salpicamiento subsuperficial se debe al sentido del flujo en la salida, el cual es paralelo al fondo de la caja de metal similar al escurrimiento subsuperficial encontrado en campo. La figura 3 muestra un esquema de los diferentes flujos en la caja de pruebas del pavimento.

Los flujos volumétricos (tasas) de salida son: escurrimiento superficial, $Q_{\text {superficial }}$, escurrimiento subsuperficial, $Q_{\text {subsuperficial }} \mathrm{y}$ escurrimiento por salpicamiento, $\mathrm{Q}_{\text {salpicamiento }}$. El flujo (tasa) de entrada se debe a la precipitación, $\mathrm{Q}_{\text {lluvia. }}$. La infiltración en el pavimento es $I$ y $\Delta V / \Delta t$ es la variación del volumen de agua dentro del conjunto bloque-arena (figura 4).

Aplicando el balance de masa sobre la caja de pavimento en las siguientes etapas, se tiene:

a) En el inicio, cuando $Q_{\text {superficial }}=0$ y $Q_{\text {subsuperficial }}=0$

De la figura 4 tenemos

$\mathrm{Q}_{\text {lluvia }}=\frac{\Delta V}{\Delta t}+\mathrm{Q}_{\text {salpicamiento }}$

En la figura 5, se tiene

$$
I=\frac{\Delta V}{\Delta t}
$$

Entonces, sustituyendo la ecuación (3) en la ecuación (2) y organizando se tiene

$\mathrm{Q}_{\text {lluvia }}-\mathrm{Q}_{\text {salpicamiento }}=I$

Recordando que la infiltración $I$ inicia con un valor alto y comienza a decrecer y $Q_{\text {salpicamiento }}$ es constante. b) Cuando se inicia $Q_{\text {superficial }}$ y $Q_{\text {subsuperficial }}=0$

Así

$\mathrm{Q}_{\text {lluvia }}=\frac{\Delta V}{\Delta t}+\mathrm{Q}_{\text {salpicamiento }}+\mathrm{Q}_{\text {superficial }}$

y

$I=\frac{\Delta V}{\Delta t}$

Entonces

$\mathrm{Q}_{\text {lluvia }}-\mathrm{Q}_{\text {sappicamiento }}-\mathrm{Q}_{\text {superficial }}=I$

c) Cuando $Q_{\text {subsuperficial }} \neq 0$ no es constante y el sistema no está en equilibrio, I continua decreciente, $Q_{\text {salpicamiento }}$ constante $y$ $Q_{\text {superficial }}$ crece, así:

$\mathrm{Q}_{\text {lluvia }}=\frac{\Delta V}{\Delta t}+\mathrm{Q}_{\text {salpicamiento }}+\mathrm{Q}_{\text {superficial }}+\mathrm{Q}_{\text {subsuperficial }}$

$\mathrm{y} \quad I=\frac{\Delta V}{\Delta t}+\mathrm{Q}_{\text {subsuperficial }}$

Entonces

$\mathrm{Q}_{\text {lluvia }}-\mathrm{Q}_{\text {salpicamiento }}-\mathrm{Q}_{\text {superficial }}=I$ 


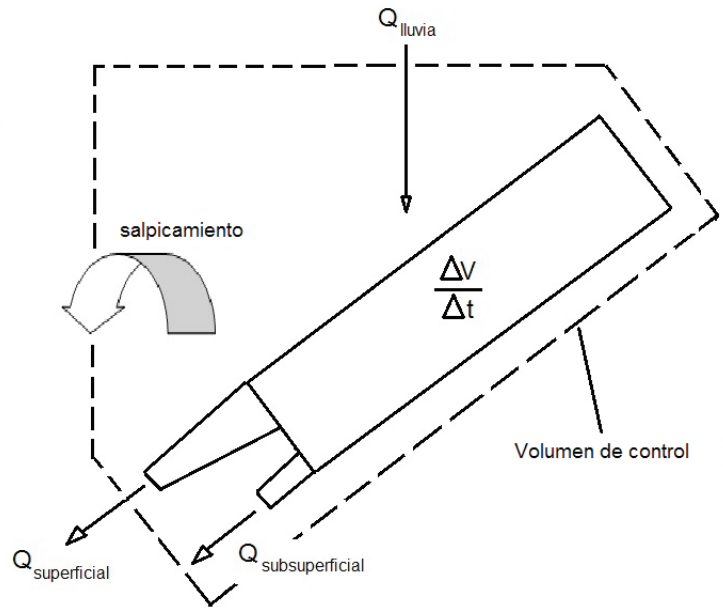

a)

Figura 4. Aplicación del volumen de control, a) alrededor del conjunto caja metálica y bloques, b) en la superficie del bloque, envolviendo el conjunto bloque-arena

d) Cuando $Q_{\text {subsuperficial }}$ y $Q_{\text {superficial }}$ son constantes, tenemos que $\frac{\Delta V}{\Delta t}=0$

Entonces

$$
Q_{\text {lluvia }}=Q_{\text {salpicamiento }}+Q_{\text {superficial }}+Q_{\text {subsuperficial }}
$$

$\mathrm{y}$

$I=\mathrm{Q}_{\text {subsuperficial }}$

De esta forma, $Q_{\text {salpicamiento }}$ puede ser calculada por la ecuación 13

$\mathrm{Q}_{\text {lluvia }}-\mathrm{Q}_{\text {superficial }}-I=\mathrm{Q}_{\text {salpicamiento }}$

Se sabe que $\mathrm{Q}_{\text {salpicamiento }}$ es constante en todas las etapas mencionadas, entonces, sustituyendo el valor de $Q_{\text {salpi- }}$ camiento, calculado en la ecuación (13), en las ecuaciones (4), (7), (10) y conociendo $\mathrm{Q}_{\text {superficial' }}$ se puede determinar la variación de $I$ en las diferentes etapas del escurrimiento.

La caja de pavimento se sometió a una lluvia simulada de una hora para cada ensayo, con intervalo entre ensayos de una semana, debido al proceso de montaje del pavimento y el secado de los bloques empleados. Se realizaron tres ensayos para cada intensidad (76.9 y $117.7 \mathrm{~mm} / \mathrm{h})$ y pendiente $(1,3$ y $5 \%)$, sumando un total de dieciocho ensayos.

\section{Análisis de los resultados}

Las tablas 2, 3 y 4 presentan los resultados de los ensayos realizados con pendientes de 1,2 y $3 \%$, respectivamente.

El $Q_{\text {lluvia }}$ se encontró multiplicando la intensidad $i$ $(\mathrm{mm} / \mathrm{h})$ medida antes del ensayo por la proyección horizontal del área del pavimento en la caja de ensayos $\left(\mathrm{A}_{\text {horizontal }}\right)$. El $\mathrm{Q}_{\text {subsuperficial }}$ se determinó por el método de medición volumétrica del caudal del flujo colectado por el tubo de drenaje instalado en la parte inferior de la caja de ensayo (figura 3), con intervalos de tiempo de colecta de 2 min y 30 seg en la primera media hora y cada $5 \mathrm{~min}$ en la segunda media hora. En el cálculo de $\mathrm{Q}_{\text {superficial }}$ también se empleó el método de medición volumétrico en el escurrimiento colectado en la canaleta superior instalada en la parte frontal de la caja (figura 4). Los valores de $Q_{\text {subsuperficial }}$ y $Q_{\text {superficial }}$ de las tablas 2, 3 y 4 se refieren a los escurrimientos subsuperficial y superficial en régimen permanente. El flujo de salida por salpicamiento $\left(\mathrm{Q}_{\text {salpicamiento }}\right)$ fue determinado con la ecuación (10), en la etapa donde los flujos de escurrimiento -superficial y subsuperficial- se encuentran en régimen permanente. El coeficiente $C$ se calcula por la razón entre $\mathrm{Q}_{\text {superficial }}$ y $\mathrm{Q}_{\text {lluvia }}$.

El término $Q_{\text {superficialtsalpicamiento }}$ es la suma de $Q_{\text {superficial }} y$ $\mathrm{Q}_{\text {salpicamiento. }}$ El coeficiente $\mathrm{C}_{\text {superficietsalpicamiento }}$ se define por la división de $\mathrm{Q}_{\text {superficial+salpicamiento }}$ entre $\mathrm{Q}_{\text {lluvia }}$. Como los ensayos fueron realizados en una caja de $50 \times 50 \mathrm{~cm}$ y considerando esta área de ensayo como un área base, entonces para analizar áreas mayores se puede suponer 
que estas áreas están constituidas por el acoplamiento (suma) de varias áreas base de $50 \times 50 \mathrm{~cm}$. Entonces, buena parte del $Q_{\text {salpicamiento }}$ de un área base pasaría para la otra área base vecina de forma concatenada. Entonces, el $\mathrm{C}_{\text {superficietsalpicamiento }}$ representa el máximo valor posible del coeficiente de escurrimiento.

La tabla 2, para pendiente de 1\%, muestra que el inicio del escurrimiento superficial sucede tiempo después de iniciado el ensayo, esto sugiere que en la etapa inicial el conjunto bloque-arena absorbe por infiltración y percolación parte del escurrimiento precipitado, considerando que la otra parte del volumen precipitado salió de la caja de ensayo en forma de salpicamiento. El escurrimiento superficial inicia cuando se satura la capa localizada en la parte superior del conjunto bloque-arena, restringiendo la infiltración y dando inicio al escurrimiento superficial.

El escurrimiento subsuperficial se inicia algunos minutos después de que inicia el escurrimiento superficial, el retardo en la aparición del escurrimiento se debe a la percolación del agua en la parte interior del conjunto bloque-arena.

Comparando los resultados de los valores medios para las intensidades $76.9 \mathrm{~mm} / \mathrm{h}$ y $117.7 \mathrm{~mm} / \mathrm{h}$, se observa que existe una ligera reducción del tiempo de inicio del escurrimiento (superficial y subsuperficial) debido al incremento de la precipitación y existe un incremento en los valores de flujo de salida (subsuperficial, superficial y salpicamiento) con el incremento de la intensidad. En el caso del coeficiente $\mathrm{C}$ existe poco aumento.

Los resultados para pendiente de 3\% (tabla 3) muestran la diminución del tiempo de inicio de escurrimien- to superficial y subsuperficial con el incremento de la intensidad, así como el ligero aumento de los valores de flujo de salida (subsuperficial, superficial e salpicamiento). Para el coeficiente $\mathrm{C}$, se observa poco aumento de este valor.

En los resultados de la tabla 4 para pendiente de 3\% también existe la diminución de los tiempos de inicio de escurrimiento superficial y subsuperficial con incremento de la intensidad, con el ligero aumento de los valores de flujo de salida (subsuperficial, superficial y salpicamiento). Para el coeficiente $\mathrm{C}$, se observa una ligera reducción de este valor.

En las tablas 2, 3 y 4 los valores de $Q_{\text {supericial }}$ para cada ensayo varían directamente con los diferentes valores de las intensidades correspondientes, esto es $Q_{\text {lluvia }}$. En consecuencia, los valores de $C$ también acompañan estas variaciones, presentando valores en la faja de $0.53 \mathrm{y}$ 0.67 , pero los valores individuales presentaron poca variabilidad entre sí. El valor medio del coeficiente de escurrimiento fue $C=0.61 \mathrm{y}$ es semejante al valor de $\mathrm{C}=0.6$ para pavimento de bloques rectangulares e inferiores al valor $\mathrm{C}=0.78$ para pavimento con bloques de concreto. Por otro lado, el $\mathrm{C}_{\text {superficialtsalpicamiento }}$ presentó valores en la faja de 0.76 a 0.89 , y se encontró cerca al del bloque de cemento e inferior al coeficiente para concreto $(C=0.95)$. De esta manera, el pavimento de bloque de solo-cemento se puede considerar como semipermeable o casi impermeable. Se sabe que por cuestiones de camino del escurrimiento en la superficie, por pérdidas de evaporación, por depresiones en el área y otros factores, el coeficiente será mucho menor que 0.95 y puede estar próximo al del coeficiente de bloque de cemento o en el mejor de los casos, cerca del coeficiente de

Tabla 2. Resultados de dos ensayos realizados con pendiente $1 \%, \mathrm{~A}_{\text {horizontal }}$ de $249.987 \mathrm{~mm}^{2}$ e intensidades de $76.9 \mathrm{~mm} / \mathrm{h}$ y $117.7 \mathrm{~mm} / \mathrm{h}$

\begin{tabular}{|c|c|c|c|c|c|c|c|c|}
\hline Pendiente $1 \%$ & & $\mathrm{i}_{\text {estimada }}=76$ & $\mathrm{~mm} / \mathrm{h}$ & & & $\mathrm{i}_{\text {estimada }}=$ & $7.7 \mathrm{~mm} / \mathrm{h}$ & \\
\hline Núm. de ensayo & Ensayo 1 & Ensayo 2 & Ensayo 3 & Media & Ensayo 1 & Ensayo 2 & Ensayo 3 & Media \\
\hline Fecha & 29/mar/11 & 29/mar/11 & $31 / \mathrm{mar} / 11$ & & 05/abr/11 & 05/abr/11 & 07/abr/11 & \\
\hline $\begin{array}{l}\text { Inicio del escurrimiento } \\
\text { superficial (min) }\end{array}$ & 02:55 & 02:41 & 02:50 & 02:48 & 01:30 & 02:04 & 02:00 & 01:51 \\
\hline $\begin{array}{l}\text { Inicio del escurrimiento } \\
\text { subsuperficial (min) }\end{array}$ & 07:53 & $11: 20$ & $10: 12$ & 09:48 & 11:00 & 07:40 & $10: 25$ & 09:41 \\
\hline $\mathrm{i}(\mathrm{mm} / \mathrm{h})$ & 76.9 & 76.9 & 75.7 & 76.50 & 113.2 & 117.4 & 111.8 & 114.13 \\
\hline $\mathrm{Q}_{\text {lluvia }}(\mathrm{l} / \mathrm{h})$ & 19.2 & 19.2 & 18.9 & 19.10 & 30.0 & 30.5 & 29.0 & 29.83 \\
\hline $\mathrm{Q}_{\text {subsuperficial }}(\mathrm{l} / \mathrm{h})$ & 1.4 & 1.2 & 1.3 & 1.30 & 1.9 & 2.2 & 1.8 & 1.97 \\
\hline $\mathrm{Q}_{\text {superficial }}(1 / \mathrm{h})$ & 11.1 & 11.8 & 11.3 & 11.40 & 18.9 & 17.8 & 18.9 & 18.53 \\
\hline $\mathrm{Q}_{\text {salpicamiento }}(\mathrm{l} / \mathrm{h})$ & 5.3 & 5.1 & 5.0 & 5.13 & 7.4 & 8.3 & 6.5 & 7.40 \\
\hline $\mathrm{C}$ & 0.60 & 0.64 & 0.62 & 0.62 & 0.66 & 0.60 & 0.67 & 0.64 \\
\hline $\mathrm{Q}_{\text {superficial+salpicamiento }}$ & 16.4 & 16.9 & 16.3 & 16.53 & 26.3 & 26.1 & 25.4 & 25.93 \\
\hline $\mathrm{C}_{\text {superficial+salpicamiento }}$ & 0.85 & 0.88 & 0.86 & 0.87 & 0.88 & 0.86 & 0.88 & 0.87 \\
\hline
\end{tabular}


Tabla 3. Resultados de los ensayos realizados con pendiente de $3 \%, \mathrm{~A}_{\text {horizontal }}$ de $249.887 \mathrm{~mm}^{2}$ e intensidades de $76.9 \mathrm{~mm} / \mathrm{h}$ y $117.7 \mathrm{~mm} / \mathrm{h}$

\begin{tabular}{|c|c|c|c|c|c|c|c|c|}
\hline \multirow{2}{*}{$\begin{array}{l}\text { Pendiente 3\% } \\
\text { Núm. de ensayo }\end{array}$} & \multicolumn{4}{|c|}{$\mathrm{i}_{\text {estimada }}=76.9 \mathrm{~mm} / \mathrm{h}$} & \multicolumn{4}{|c|}{$\mathrm{i}_{\text {estimada }}=117.7 \mathrm{~mm} / \mathrm{h}$} \\
\hline & Ensayo 1 & Ensayo 2 & Ensayo 3 & Media & Ensayo 1 & Ensayo 2 & Ensayo 3 & Media \\
\hline Fecha & 19/abr/11 & 19/abr/11 & $21 / a b r / 11$ & & 26/abr/11 & $26 / a b r / 11$ & 28/abr/11 & \\
\hline $\begin{array}{l}\text { Inicio del escurrimiento } \\
\text { superficial (min) }\end{array}$ & 02:15 & 01:41 & 02:00 & 01:58 & $01: 57$ & 01:15 & 01:09 & 01:27 \\
\hline $\begin{array}{l}\text { Inicio del escurrimiento } \\
\text { subsuperficial (min) }\end{array}$ & $15: 01$ & 09:00 & $16: 15$ & $13: 25$ & 08:57 & 09:06 & 07:30 & 08:31 \\
\hline $\mathrm{i}(\mathrm{mm} / \mathrm{h})$ & 80.9 & 76.4 & 76.0 & 77.77 & 113.7 & 124.3 & 119.4 & 119.13 \\
\hline $\mathrm{Q}_{\text {lluvia }}(\mathrm{l} / \mathrm{h})$ & 20.2 & 19.1 & 19.0 & 19.43 & 28.4 & 31.1 & 29.8 & 29.77 \\
\hline$Q_{\text {subsuperficial }}(1 / h)$ & 1.1 & 2.5 & 1.1 & 1.57 & 1.9 & 3.5 & 3.5 & 2.97 \\
\hline $\mathrm{Q}_{\text {superficial }}(\mathrm{l} / \mathrm{h})$ & 11.3 & 9.9 & 10.5 & 10.57 & 17.6 & 17.5 & 17.6 & 17.57 \\
\hline $\mathrm{Q}_{\text {salpicamiento }}(\mathrm{l} / \mathrm{h})$ & 6.7 & 4.2 & 6.2 & 5.70 & 7.0 & 6.5 & 5.1 & 6.20 \\
\hline C & 0.58 & 0.53 & 0.58 & 0.56 & 0.64 & 0.58 & 0.60 & 0.61 \\
\hline$Q_{\text {superficial+salpicamiento }}$ & 18.0 & 14.1 & 16.7 & 16.3 & 24.6 & 24.0 & 22.7 & 23.8 \\
\hline $\mathrm{C}_{\text {superficial+salpicamiento }}$ & 0.89 & 0.74 & 0.88 & 0.84 & 0.87 & 0.77 & 0.76 & 0.80 \\
\hline
\end{tabular}

Tabla 4. Resultados de los ensayos realizados con pendiente de $5 \%, \mathrm{~A}_{\text {horizontal }}$ de $249.687 \mathrm{~mm}^{2}$ e intensidades de $76.9 \mathrm{~mm} / \mathrm{h}$ y $117.7 \mathrm{~mm} / \mathrm{h}$

\begin{tabular}{|c|c|c|c|c|c|c|c|c|}
\hline Pendiente 5\% & & $\mathrm{i}_{\text {estimada }}=76$ & $\mathrm{~mm} / \mathrm{h}$ & & & $\mathrm{i}_{\text {estimada }}=117$ & $7 \mathrm{~mm} / \mathrm{h}$ & \\
\hline Núm. de ensayo & Ensayo 1 & Ensayo 2 & Ensayo 3 & Media & Ensayo 1 & Ensayo 2 & Ensayo 3 & Media \\
\hline Fecha & $22 / \mathrm{mar} / 11$ & $22 / \mathrm{mar} / 11$ & $24 / \mathrm{mar} / 11$ & & 15/mar/11 & $15 / \mathrm{mar} / 11$ & 17/mar/11 & \\
\hline $\begin{array}{l}\text { Inicio del escurrimiento } \\
\text { superficial (min) }\end{array}$ & 02:07 & $02: 11$ & $01: 43$ & 02:00 & 01:25 & 01:04 & 01:40 & $01: 23$ \\
\hline $\begin{array}{l}\text { Inicio del escurrimiento } \\
\text { subsuperficial (min) }\end{array}$ & $12: 30$ & $14: 14$ & $14: 12$ & $13: 38$ & $11: 30$ & 08:04 & 09:42 & 09:45 \\
\hline $\mathrm{i}(\mathrm{mm} / \mathrm{h})$ & 77.64 & 75.52 & 76.23 & 76.46 & 122.22 & 117.03 & 119.86 & 119.70 \\
\hline $\mathrm{Q}_{\text {lluvia }}(\mathrm{l} / \mathrm{h})$ & 19.4 & 18.9 & 19.0 & 19.10 & 30.5 & 29.2 & 29.9 & 29.87 \\
\hline$Q_{\text {subsuperficial }}(1 / \mathrm{h})$ & 1.3 & 2.1 & 2.1 & 1.83 & 2.6 & 2.9 & 2.7 & 2.73 \\
\hline $\mathrm{Q}_{\text {superficial }}(\mathrm{l} / \mathrm{h})$ & 11.3 & 10.8 & 11.2 & 11.10 & 18.4 & 16.8 & 17.2 & 17.47 \\
\hline $\mathrm{Q}_{\text {salpicamiento }}(\mathrm{l} / \mathrm{h})$ & 5.4 & 3.9 & 3.6 & 4.30 & 6.8 & 6.7 & 7.4 & 6.97 \\
\hline C & 0.62 & 0.61 & 0.63 & 0.62 & 0.61 & 0.59 & 0.59 & 0.60 \\
\hline$Q_{\text {superficial }+ \text { salpicamiento }}$ & 16.7 & 14.7 & 14.8 & 15.4 & 25.2 & 23.5 & 24.6 & 24.43 \\
\hline $\mathrm{C}_{\text {superficiallsalpicamiento }}$ & 0.86 & 0.78 & 0.78 & 0.81 & 0.83 & 0.80 & 0.82 & 0.82 \\
\hline
\end{tabular}

bloque de adoquines. Debido a esta incertidumbre, un estudio de campo de un área mayor que la estudiada se encuentra en desarrollo.

Las figuras 5 y 6 presentan la curva de la variación temporal del escurrimiento superficial con pendientes de 1,3 y 5\%, y sometidos a lluvias de intensidades 76.9 $\mathrm{mm} / \mathrm{h}$ y $117.7 \mathrm{~mm} / \mathrm{h}$, respectivamente.

En la figura 5 el ascenso de las curvas sucede hasta el tiempo de 30 minutos, después las curvas presentan valores constantes, acompañando la horizontal.
Las curvas de la figura 8 presentan un incremento más corto, estabilizándose 20 min después de iniciados los ensayos. Comparando las figuras 5 y 6 , se observa que las curvas de escurrimiento superficial para intensidad $76.9 \mathrm{~mm} / \mathrm{h}$ presenta un intervalo de ascenso mayor que la curva para intensidad $117.7 \mathrm{~mm} / \mathrm{h}$, esto se debe al aumento de la intensidad. En las figuras 5 y 6, las curvas presentan un comportamiento oscilante, el escurrimiento en la curva con pendiente de $1 \%$ o mayor a las de 3 y $5 \%$, pero la curva del escurrimiento con $5 \%$ es li- 
geramente superior a la de $3 \%$, para intensidad 76.9 $\mathrm{mm} / \mathrm{h}$ (figura 7). En la figura 6 las curvas de 3 y $5 \%$ casi se sobreponen.

Las figuras 7 y 8 presentan la curva de variación temporal del escurrimiento subsuperficial para ensayos realizados con pendientes de 1,3 y $5 \%$, y sometidos a lluvias de intensidades de $76.9 \mathrm{~mm} / \mathrm{h}$ y $117.7 \mathrm{~mm} / \mathrm{h}$.

Se observa que la ascensión de las curvas suceden en el intervalo de tiempo de $10 \mathrm{~min}$ a 30 min después de iniciados los ensayos. Luego los valores oscilan entorno de la horizontal.

En la figura 7 las curvas suben en el intervalo de 7 a 20 min después del inicio de los ensayos, luego los valores oscilan en la horizontal. En la comparación de las curvas (figuras 7 y 8) se comprueba el efecto del aumento en la intensidad sobre el tiempo de ascenso de las curvas.

Existen cambios en las curvas debido a la pendiente, con el aumento de la pendiente se incrementan los flujos de escurrimiento subsuperficial, este incremento es más visible en las curvas con intensidad de $117.7 \mathrm{~mm} / \mathrm{h}$ (figura 10). Por otro lado, comparando los valores medios de $Q_{\text {subsuperficial }}$ de las tablas 2, 3 y 4, para la intensidad de $76.9 \mathrm{~mm} / \mathrm{h}$, se constata que para $1 \%$ de pen- diente el valor de $\mathrm{Q}_{\text {subsuperficial }}$ fue cerca de $20 \%$ menor que el de pendiente de $3 \%$, y $40 \%$ menor que el de pendiente de $5 \%$. Eso puede ocurrir en función de que el tubo de drenaje esté localizado en la parte inferior, del lado donde se encuentra la canaleta colectora del escurrimiento superficial, entonces, conforme se inclina la caja metálica de ensayo, la tendencia a que el agua se concentre en esa región favorece el drenaje en este punto (figura 4). Cuando se compara la variación de la tasa de escurrimiento subsuperficial entre las pendientes $3 \mathrm{y}$ $5 \%$, se muestra que el incremento del escurrimiento subsuperficial es casi $18 \%$. En el caso de los valores de escurrimiento subsuperficial para intensidad de 117.7 $\mathrm{mm} / \mathrm{h}$ se comprueba que con pendiente de $1 \%$ escurre menos que con pendientes 3 y $5 \%$, pero el $Q_{\text {subsuperficial }}$ de la pendiente $5 \%$ es menor que el de $3 \%$, se puede atribuir este comportamiento a varios factores, pues en algunos ensayos hubo una oscilación significativa de los resultados, los factores serían: variabilidad de las intensidades en cada ensayo; formas de asentamiento de los bloques; aumento de resistencia (pérdidas) en tubo de drenaje con el aumento del flujo del escurrimiento por percolación debido a la variación de la pendiente y al incremento de la intensidad (flujo de entrada), y au-

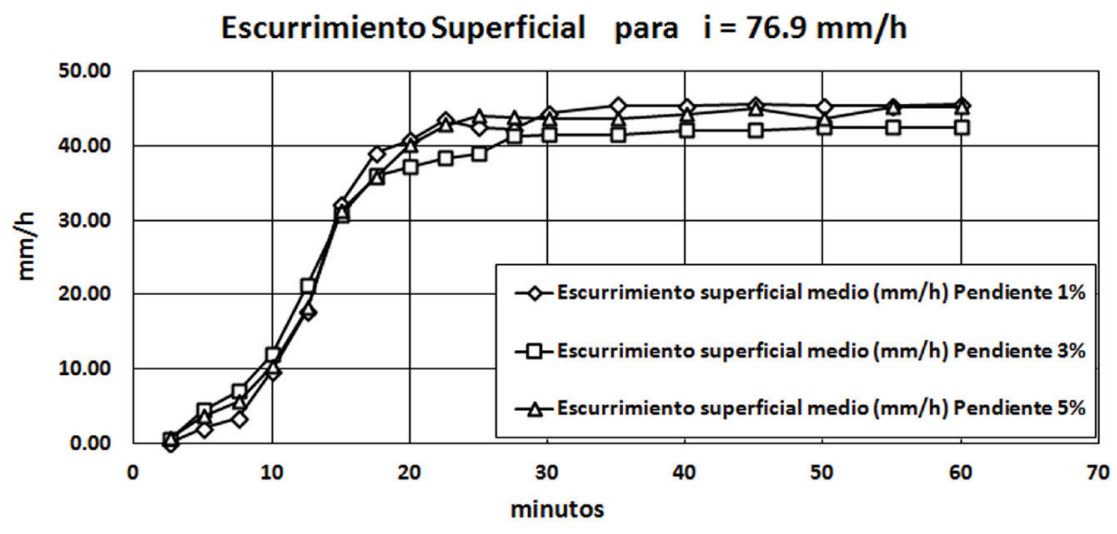

Escurrimiento Superficial para $\mathrm{i}=\mathbf{1 1 7 . 7} \mathbf{\mathrm { mm }} / \mathrm{h}$

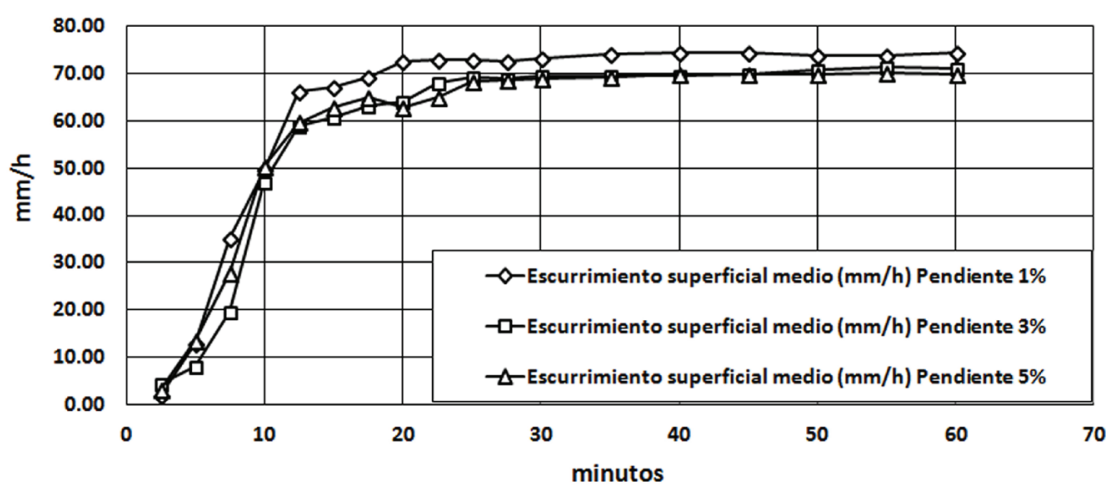

Figura 5. Curvas medias del escurrimiento superficial en pavimento con diferentes pendientes $i=76.9$ $\mathrm{mm} / \mathrm{h}$
Figura 6. Curvas de las medias del escurrimiento superficial en pavimento con diferentes pendientes $i=117.7$ $\mathrm{mm} / \mathrm{h}$ 

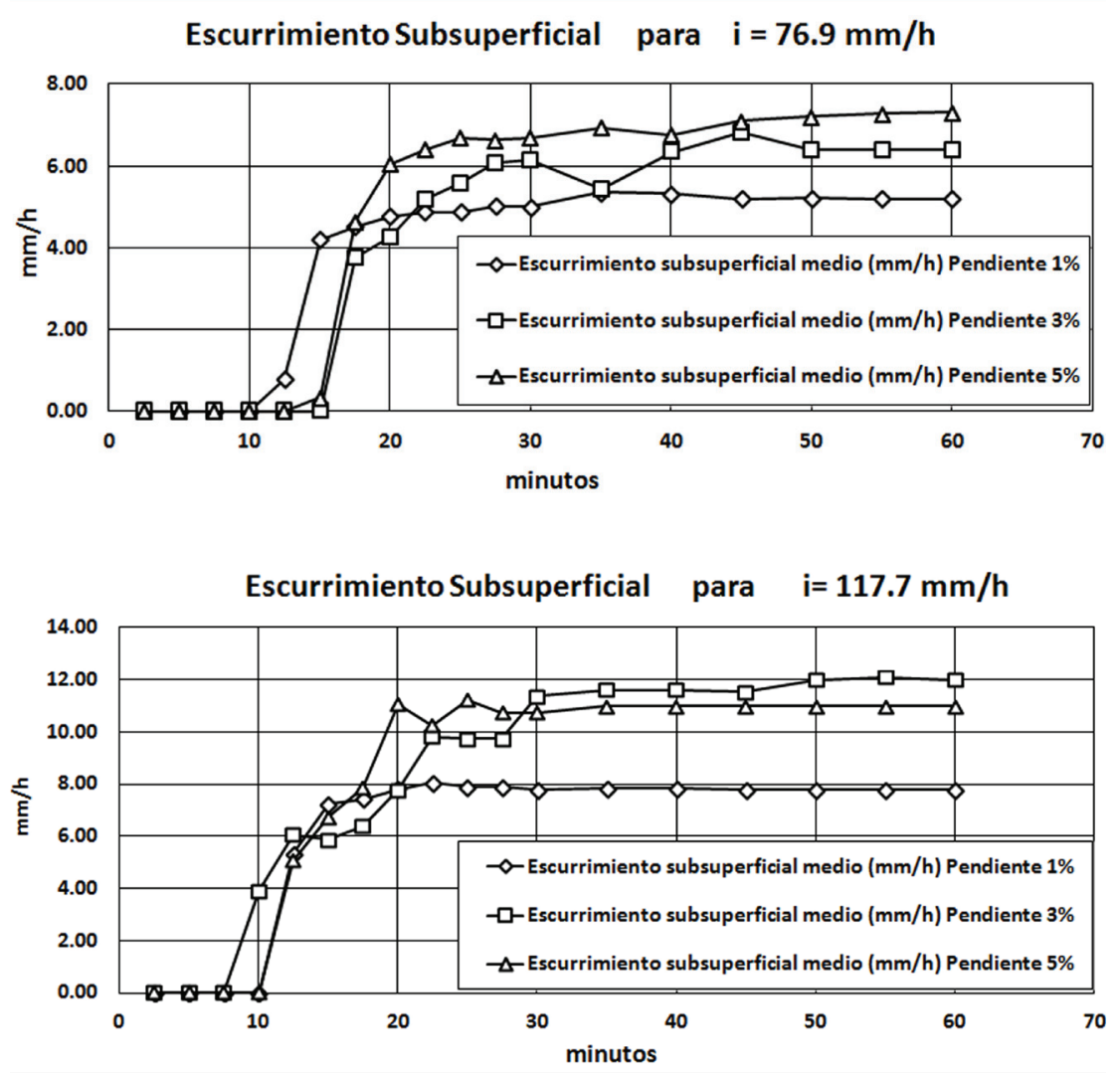

Figura 7. Curvas de las medias del escurrimiento subsuperficial en pavimento con diferentes pendientes $i=76.9 \mathrm{~mm} / \mathrm{h}$

Figura 8. Curvas de las medias del escurrimiento subsuperficial en pavimento con diferentes pendientes $i=117.7 \mathrm{~mm} / \mathrm{h}$

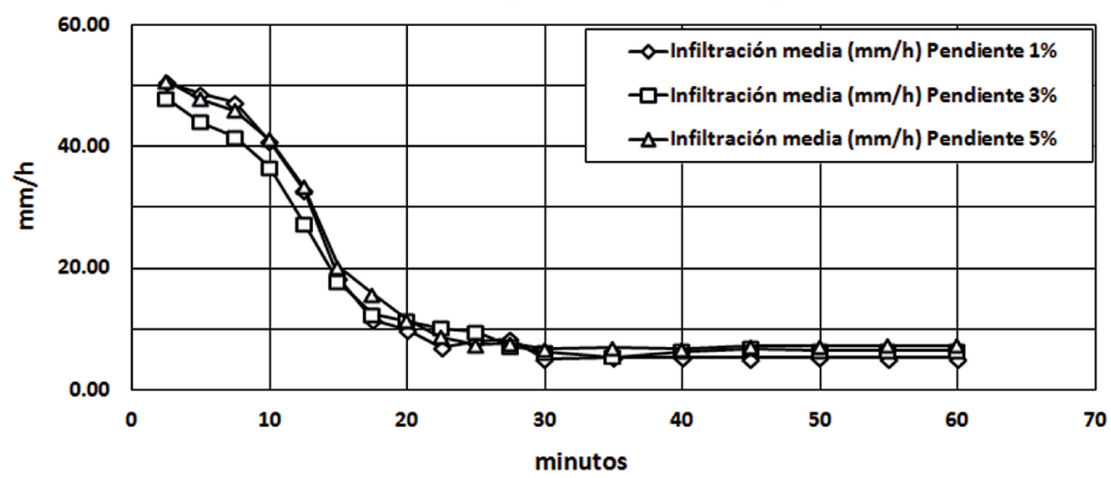

Figura 9. Curvas medias de la infiltración en pavimento con diferentes pendientes $i=76.9 \mathrm{~mm} / \mathrm{h}$ mento del escurrimiento superficial debido a la acción de la fuerza de la gravedad (variación de la pendiente). Este fenómeno coincide con Marchioni y Silva (2010) que recomiendan la construcción de pavimentos con el máximo de $5 \%$ de pendiente, pues usar valores superiores aumenta el escurrimiento superficial en decremento del escurrimiento subsuperficial o de la infiltración.

En la implantación en campo de los pavimentos semipermeables varios aspectos pueden mejorar o perjudicar significativamente el escurrimiento subsuperficial:
- La humedad del suelo, disminuye la capacidad de percolación del suelo generando un escurrimiento subsuperficial paralelo a la superficie de los bloques.

- La conductividad del suelo, mayor conductividad favorece la percolación vertical del agua disminuyendo el escurrimiento subsuperficial en las capas debajo de los bloques. 


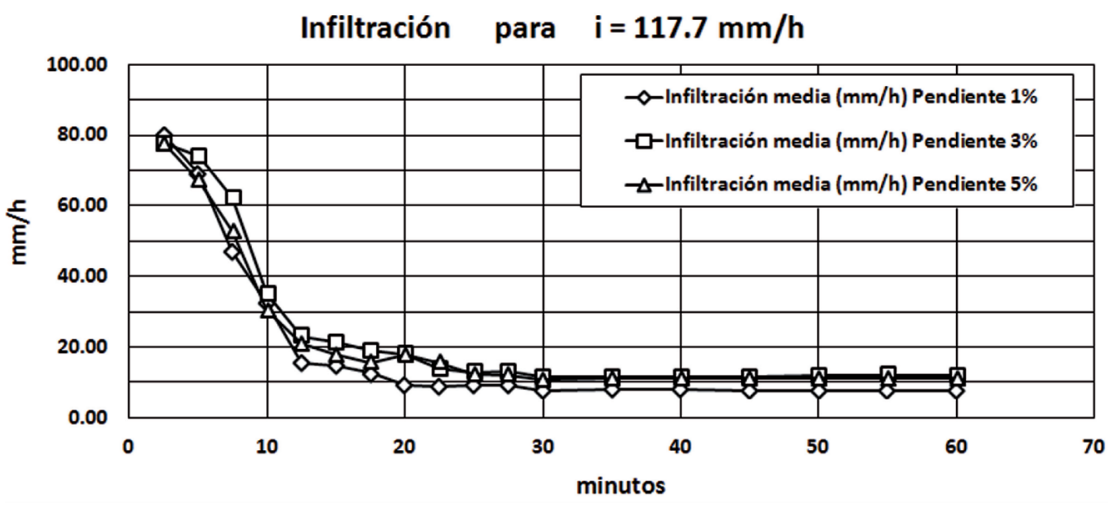

Figura 10. Curvas de las medias de la infiltración en pavimento con diferentes pendientes $i=117.7 \mathrm{~mm} / \mathrm{h}$
- La utilización de tubos de drenaje en la base/subbase, a fin de colectar parte del escurrimiento subsuperficial y permitir el aumento de la infiltración.

Las figuras 9 y 10 presentan las curvas de la variación temporal de la infiltración para ensayos realizados con pendientes de 1,3 y $5 \%$, y sometidos a lluvias de intensidades de $76.9 \mathrm{~mm} / \mathrm{h}$ y $117.7 \mathrm{~mm} / \mathrm{h}$.

La figura 10 muestra las curvas de infiltración con comportamiento coherente donde las curvas de infiltración se obtuvieron experimentalmente en campo para diferentes tipos de suelos. Las curvas presentan una caída continua en los primeros $30 \mathrm{~min}$, luego la infiltración se estabiliza presentando valores casi constantes.

En la figura 10 los valores de las tasas de infiltración son mayores que los presentados en la figura 9, corroborando el efecto del aumento de la intensidad en el escurrimiento.

Se debe aclarar que los comportamientos de las curvas de infiltración y del escurrimiento subsuperficial tienen una relación directa, relación que se establece a partir del balance de masa en el conjunto bloque-arena (figura 4), que se expresa en las ecuaciones (9) y (12).

\section{Conclusiones}

El coeficiente de escurrimiento medio para el pavimento de bloque suelo-cemento, considerando solo el escurrimiento superficial, fue $C=0.61$ y es semejante al valor $\mathrm{C}=0.6$ para pavimento de bloques rectangulares e inferior al valor $C=0.78$ para pavimento con bloque de concreto. Por otro lado, cuando consideramos el salpicamiento, se tiene el coeficiente $\mathrm{C}_{\text {superficial salpicamiento }}$ que presentó valores en la faja de 0.74 a 0.89 , encontrándose cerca del coeficiente del bloque de cemento que es inferior al coeficiente para concreto $(C=0.95)$. Entonces, el pavimento de bloque de suelo-cemento puede considerarse semipermeable.
Referente al comportamiento de las curvas de variación temporal (tasas) de los escurrimientos (superficial, subsuperficial e infiltración) se observa lo siguiente:

- Con el incremento de la intensidad también crecen los valores de las tasas de escurrimiento (superficial, subsupeficial e infiltración).

- Para una intensidad constante y un incremento de la pendiente de $1 \%$ a $3 \%$ se observó una reducción de las tasas de escurrimiento superficial. Cuando el incremento de la pendiente es de $3 \%$ a $5 \%$ se observa un aumento en la tasa de escurrimiento superficial, confirmando la recomendación de Marchioni y Silva (2010) que mencionan la restricción del uso de pavimentos para pendientes iguales o superiores a $5 \%$ debido al incremento del escurrimiento superficial.

El empleo de una caja de ensayo de $50 \times 50 \mathrm{~cm}$ oculta ciertos factores de escala, el uso de mayores áreas de ensayo determina mayor influencia de factores como: aumento del tiempo de concentración debido a la mayor distancia recorrida por el escurrimiento en la superficie; reducción del volumen de salpicamiento perdido en las laterales del área de ensayo y por consiguiente un aumento del escurrimiento superficial; pérdidas por evaporación; aumento de la acción de la rugosidad del material de los bloques sobre escurrimiento; irregularidades en el asentamiento de los bloques, lo que genera depresiones sobre la superficie de ensayo lo que aumenta su capacidad de detención y resistencia al escurrimiento; y otros. Los factores mencionados determinarán que el coeficiente de escurrimiento pueda ser menor que 0.95 y estar próximo al del coeficiente de bloque de cemento o, en el mejor de los casos, cerca del coeficiente del bloque rectangular. Con la finalidad de evaluar estos factores se encuentra en desarrollo un estudio de campo con un área mayor de estudio. 


\section{Agradecimentos}

Agradecemos al Conselho Nacional de Desenvolvimento Científico e Tecnológico (CNPq) y a la Financiadora de Estudos e Projetos (FINEP) por el apoyo financiero al proyecto de investigación en desenvolvimiento y por la beca de iniciación científica concedida al Sr. Jeferson Santos de Brito, pues fueron fundamentales para el desarrollo de las actividades.

\section{Referencias}

Andersen C.T, Foster I.D.L., Pratt C.J. Role of Urban Surfaces (Permeable Pavements) in Regulating Drainage and Evaporation: Development of a Laboratory Simulation Experiment. Hydrological Processes, volumen 13 (número 4), 1999: 597.

Araújo P.R., Tucci C.E.M., Goldenfum J.A. Avaliação da Eficiência dos Pavimentos Permeáveis na Redução de Escoamento Superficial. Revista Brasileira de Recursos Hídricos RBRH, volumen 5 (número 3), julio-septiembre de 2000: 21-29.

Armfield. Manual de uso do simulador de chuvas, Armfield, Inglaterra, 1998.

Brandão C.P. Verificação da Viabilidade de Aplicação de Bloco de Solo-Cimento em Pavimento, monografía de conclusión de carrera, Brasil, Universidade Católica do Salvador, 2010.

Cardoso L.H.S. Avaliação do Potencial Erosivo dos Solos de Maior Ocorrência em Áreas de Encosta da Cidade de Salvador/Bahia, monografía de conclusión de carrera, Brasil, Universidade Católica do Salvador, 2007.

Hunt W.F. y Collins K.E. Permeable Pavement: Research Update and Design Implications, Raleigh, NC. Boletín de la North Caroline Extension Service, 2008, 12p.
James W. y Von-Langsdorff H. The Use of Permeable Concrete Block Pavement in Controlling Enviromental Stressors in Urban Areas, International Conference on Concrete Block Paving (PAVE) $\left(7^{\text {th }}, 12^{\text {th }}-17^{\text {th }}\right.$ octubre, 2003 , Sun City, Sur de Africa). Document Transformation Technologies, Sun City, Sur de Africa, Conference Planners, 2003.

Marchioni M. y Silva C.O. Cartilla de pavimento intertravado permeável, melhores práticas, São Paulo, Associação Brasileira de Cimento Portland (ABCP), 2010, 24 p.

Matos J.E.R. Chuvas Intensas na Bahia, Equações e Metodologias de Regionalização, 1a ed., Salvador, Brasil, Editora de la Universidade Federal da Bahia EDUFBA, 2006.

Silveira A.L.L. Hidrologia urbana no Brasil, en: Braga B., Tucci C.E.M. y Tozzi M., 1998, Drenagem Urbana, Gerenciamento, Simulação, Controle, 3a ed., Porto Alegre, Brasil, Editora de la Universidade Federal do Rio Grande do Sul, 1998

Stormwater-Virginia D.C.R.. Permeable Pavement, Design Specification Núm. 7, Virgina, VA DCR, Version 1.8, March 1, 2011.

\section{Este artículo se cita:}

\section{Citación estilo Chicago}

Zegarra-Tarqui, Jorge Luis, Jeferson Santos de Brito, Miriam de Fatima-Carvalho. Escurrimiento en pavimentos de bloques de suelo-cemento: un abordaje experimental. Ingeniería Investigación y Tecnología, XVI, 01 (2015): 35-47.

\section{Citación estilo ISO 690}

Zegarra-Tarqui J.L., Santos de Brito J., De Fátima-Carvalho M. Escurrimiento en pavimentos de bloques de suelo-cemento: un abordaje experimental. Ingeniería Investigación y Tecnología, volumen XVI (número 1), enero-marzo 2015: 35-47.

\section{Semblanza de los autores}

Jorge Luis Zegarra-Tarqui. Ingeniero mecánico de fluidos por la Universidad Mayor de San Marcos, Perú (1992). Maestro en ingeniería mecánica por la Universidad Federal de Uberlândia, Brasil (1996) y doctor en recursos hídricos y saneamiento ambiental por el Instituto de Pesquisas Hidráulicas (IPH), Brasil (2001). Actualmente es profesor de la Universidad Federal de Minas Gerais-UFMG, imparte cursos en el área de mecánica de fluidos e hidráulica y participa en el posgrado (maestría y doctorado) en el área de recursos hídricos.

Jeferson Santos-de Brito. Ingeniero civil por la Universidad Católica del Salvador, Brasil (2011), becario de iniciación científica por el Consejo Nacional de Desenvolvimiento Científico y Tecnológico (CNPq), de 2010 a 2011. Tiene experiencia en control tecnológico de materiales de construcción, presupuestos y ejecución de refuerzo estructural. Actualmente es ingeniero Trainee en la Produman Engenharia actuando principalmente en obras de infraestructura (conservación y mantenimiento).

Miriam de Fátima-Carvalho. Ingeniera civil por la Escuela de Ingeniería Kennedy, Brasil (1991), maestra y doctora en geotecnia por la Escuela de Ingeniería de São Carlos de la Universidad de São Paulo (USP), Brasil (1994 y 1999), tiene estancia posdoctorado en la Universite Joseph Fourier-LIRIGM, Francia (2007). Actualmente es profesora e investigadora de la Universidad Católica del Salvador, Brasil, trabajando principalmente en los siguientes temas: rellenos sanitarios, geotecnia ambiental, contenciones y obras de tierra. 